\title{
THE DYNAMICS OF A PARAMETRICALLY DRIVEN DAMPED PENDULUM
}

\author{
A. DAS ${ }^{*}$ \\ Department of Mathematics, Jadavpur University \\ Kolkata-700032, INDIA \\ E-mail: adas@math.jdvu.ac.in \\ K. KUMAR \\ Department of Physics and Meteorology \\ Indian Institute of Technology \\ Kharagpur-721302, INDIA \\ E-mail: kumar@phy.iitkgp.ernet.in
}

\begin{abstract}
Ordered and chaotic states of a parametrically driven planar pendulum with viscous damping are numerically investigated. The damping makes the number of chaotic windows fewer but with larger width. Stroboscopic maps of the chaotic motion of the pendulum, driven either subharmonically or harmonically, show strange attractors with inversion symmetry in the phase plane.
\end{abstract}

Key words: parametric vibration, Floquet analysis, Lyapunov exponents, stroboscopic maps, chaos.

\section{Introduction}

The problem of parametrically driven physical system is known since the pioneering experiment of Faraday (1831), who observed subharmonically forced waves on the fluid surface under gravity modulation. The simplest possible parametrically forced system is a planar pendulum whose point of suspension is vibrated sinusoidally with amplitude $a$ and frequency $\omega$ in a vertical plane (Landau and Lifschitz, 1976). The small amplitude oscillation of the pendulum is then governed by the Mathieu equation. Floquet analysis of the Mathieu equation gives tongue shaped instability zones in $a$ - $\omega$ plane (Bender and Orszag, 1978; Jordan and Smith, 1977). The pendulum can be excited only for values of parameters ( $a$ and $\omega)$ within these zones. If the pendulum oscillates with a period twice the driving period $T(=2 / \omega)$ the pendulum is said to be excited subharmonically. On the other hand, harmonic (synchronous) excitation means the period of the oscillating pendulum is same as that of the driving. The tongues corresponding to subharmonic and harmonic responses with respect to the forcing frequency appear alternately in the $a$ - $\omega$ plane, and they never intersect each other. In the absence of damping, the pendulum can be excited with infinitesimal forcing amplitude, if the driving frequency is chosen properly. The pendulum, however, requires finite forcing above a threshold value in the presence of damping. Once the pendulum is driven, the dynamics is governed by nonlinear dynamical equations. The sequence of period-doubling bifurcation and chaos in the parametrically driven pendulum is investigated both theoretically (McLaughlin, 1981; Bartuccelli et al., 2001; Ling and Yang, 2006) as well as experimentally (Leven et al., 1985; Van de Water et al., 1991). Controlling the chaotic motion of a driven pendulum has also been studied (Starrett and Tagg, 1995). However, a systematic

\footnotetext{
* To whom correspondence should be addressed
} 
investigation of the chaotic motion of a parametrically forced pendulum is missing. The strange attractors of a parametrically driven pendulum are not investigated either.

In this paper, we have investigated numerically ordered and chaotic states of parametrically driven pendulum in the presence of viscous damping. For a given damping and forcing frequency, the pendulum shows ordered and chaotic states in several windows in the forcing amplitude $a$. We have computed Lyapunov exponents for these states to and the chaotic windows in the forcing amplitude $a$. The phase portrait of the ordered (nonlinear) state shows inversion symmetry in the phase plane for a subharmonically excited pendulum. However, the phase portrait of a harmonically excited pendulum does not show such inversion symmetry. The pendulum shows chaos always through a sequence of period doubling bifurcations. The sum of all three Lyapunov exponents is always equal to $-\Lambda$ which is equal to negative of the half of the damping coefficient. One of the Lyapunov exponents of a periodically driven pendulum is always zero, and the remaining two exponents show mirror symmetry about the $a$-axis. We have also investigated the stroboscopic maps for all values of the phase angle $\phi=\omega t$. The stroboscopic maps for all values of the phase $\phi$, show strange attractors with inversion symmetry in the phase plane for both a harmonically and subharmonically driven pendulum. The shape of these strange attractors repeats after the phase $\phi=2 \pi$.

\section{Stability of the pendulum}

The stability of a parametrically driven planar pendulum (Landau and Lifschitz, 1976) is governed by the linearized pendulum equation given by

$$
\ddot{X}+2 \Lambda \dot{X}+\omega_{0}(1-a \cos \omega t) X=0
$$

where $\omega_{0}$ is the natural frequency of the pendulum in the absence of any damping. We set without any loss of generality $\omega_{0}=1$ in the rest of the paper. The term $2 \Lambda \dot{X}$ is the phenomenological viscous damping force. We present briefly the stability of the parametrically driven pendulum with damping before we investigate its nonlinear behavior. The solution of linear damped harmonic oscillator is investigated using different techniques (Bender and Orszag, 1978). However, the Floquet analysis (see e.g., (Kumar, 1996)) is used to determine the stability of the periodically driven damped pendulum. The dimensionless angular position $X$ is expanded as

$$
X(t)=e^{(s+i \alpha \omega) t} \sum_{n=-\infty}^{\infty} X_{n} e^{i n \omega t}
$$

where $s$ is the real part of the Floquet multiplier, and $i \alpha \omega$ the imaginary part. Inserting the expression for $X(t)$ in the Eq.(2.1), we obtain a recursion relation given by

$$
A_{n} X_{n}=a\left(X_{n-1}+X_{n+1}\right)
$$

where

$$
A_{n}=2\left[\{s+i(n+\alpha) \omega\}^{2}+2 \Lambda\{s+i(n+\alpha) \omega\}+1\right] .
$$

The stability boundaries of the forced pendulum are obtained by setting $s=0$. The subharmonically excited state corresponds to $\alpha=1 / 2$ and the harmonically excited state corresponds to $\alpha=0$. We have investigated only these two cases. The recursion relation (Eq.(2.3)) is converted to a generalized eigenvalue problem (Kumar, 1996) in the form given below. 


$$
\mathcal{A} \xi=a \mathcal{B} \xi
$$

where $\mathcal{A}$ is the square diagonal matrix with complex elements $A_{n}(n=\cdots,-2,-1,0,1,2, \cdots)$. The matrix $\mathcal{B}$ is a banded square matrix defined as $\mathcal{B}_{i+1, i}=\mathcal{B}_{i, i+1}=1(i=1,2,3,4, \cdots)$. This means the first sub-diagonal and first super-diagonal elements are identical and equal to unity. All other elements are zero. The matrix $\xi$ is a column matrix, whose transpose is given by $\xi^{T}=\left(\cdots, X_{-2}, X_{-1}, X_{0}, X_{1}, X_{2}, \cdots\right)$. Real eigenvalues of the matrix $\mathcal{B}^{-1} \mathcal{A}$ are possible values of $a$, while those of $\mathcal{A}^{-1} \mathcal{B}$ are possible values of $a^{-1}$ for a fixed value of damping $\Lambda$ and forcing frequency $\omega$. Eigenvalues with any preassigned accuracy may be obtained by suitably truncating the matrices. This corresponds to truncating the expansion of $X(t)$ at a given value of $n$. The marginal stability zones are obtained by plotting few least values of $a$ as a function of the forcing frequency $\omega$.
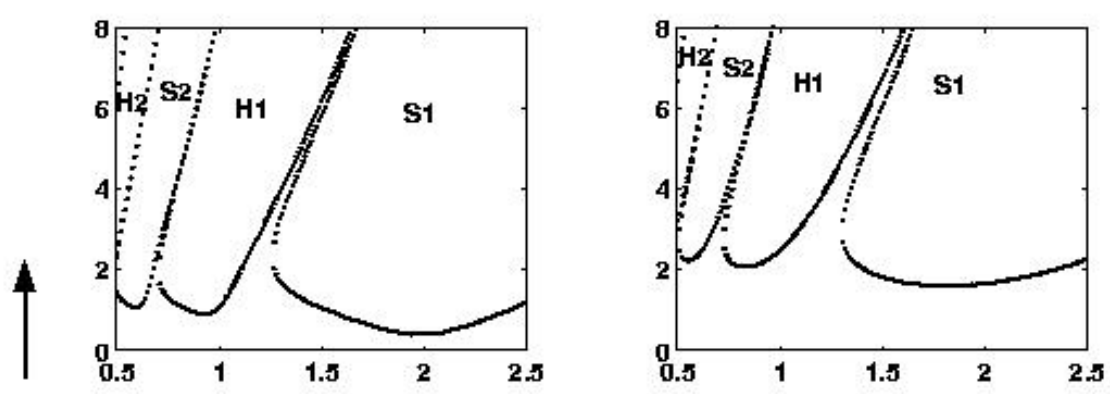

a
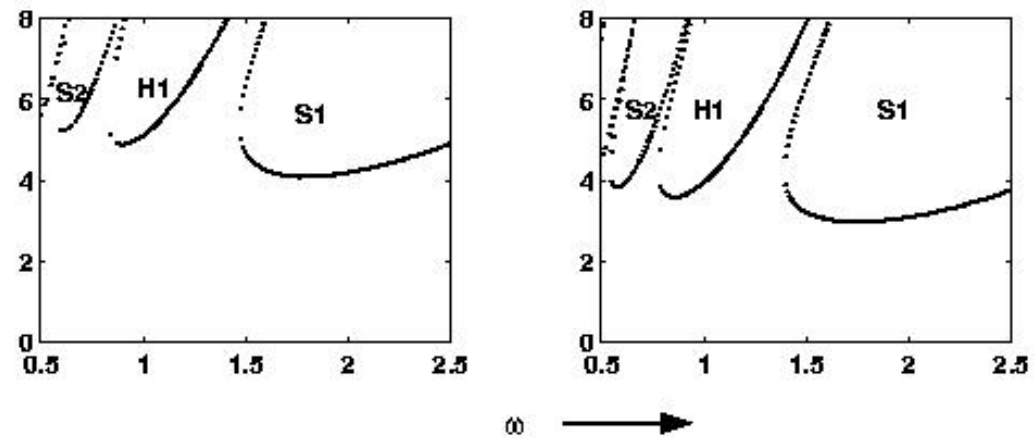

Fig.1. Marginal stability boundaries of a parametrically driven pendulum. Labels ' $\mathrm{Hn}$ ' and 'Sn' correspond to $n^{\text {th }}$ harmonic and subharmonic instability zones, respectively. Four figures (clockwise from the left top) are for the damping coefficient $\Lambda=0.1,0.4,0.7$, and $0: 9$ respectively.

Figure 1 shows the stability boundaries of a parametrically forced damped pendulum, and the effect of the viscous damping on the stability boundaries. The tongue-like zones in $a-\omega$ plane describe various regions of instabilities. If the values of $a$ and $\omega$ are chosen within any of the tongues, the fixed point corresponding to the normal equilibrium of the pendulum becomes unstable. The pendulum starts swinging. The response frequency is half the driving frequency $\omega$ for all $a$, on or inside all tongues labeled ' $\mathrm{Sn}$ ' $(n=1,2, \ldots)$. On the other hand, the response frequency is same as the forcing frequency for all $a$, on or inside the tongues labeled 'Hn' $(n=1,2, \ldots)$. These 'Sn's and 'Hn's correspond to subharmonic and harmonic responses respectively. The angular position of the pendulum starts growing and ultimately the 
nonlinearity dictates the dynamics. The angular position $X(t)$ of the pendulum shows $2 n-1$ number of maxima/minima corresponding to $n^{\text {th }}(n=1,2,3, \ldots)$ subharmonic tongue ' $\mathrm{Sn}$ ' in a period double the driving period $T=2 \pi / \omega$. Similarly, the variable $X(t)$ corresponding to $n^{\text {th }}$ harmonic tongue, labeled 'Hn' shows $n$ number of maxima/minima in period $T$. For the values of $a$ and $\omega$ outside the regions covered by any tongue, the pendulum stays at its normal equilibrium. The fixed point corresponding to the normal equilibrium is stable. In the absence of damping, all the tongues touch the $\omega$ - axis (Bender and Orszag, 1978). The pendulum can be driven with infinitesimal driving amplitude for suitably chosen driving frequencies. In the presence of damping, a finite threshold is required to drive the pendulum. The minimum values of the external drive are different for different tongues. However, the least threshold corresponds to the lowest point of the first subharmonic (S1) tongue. The curvature at the lowest points of all the tongues decreases with an increase of damping.
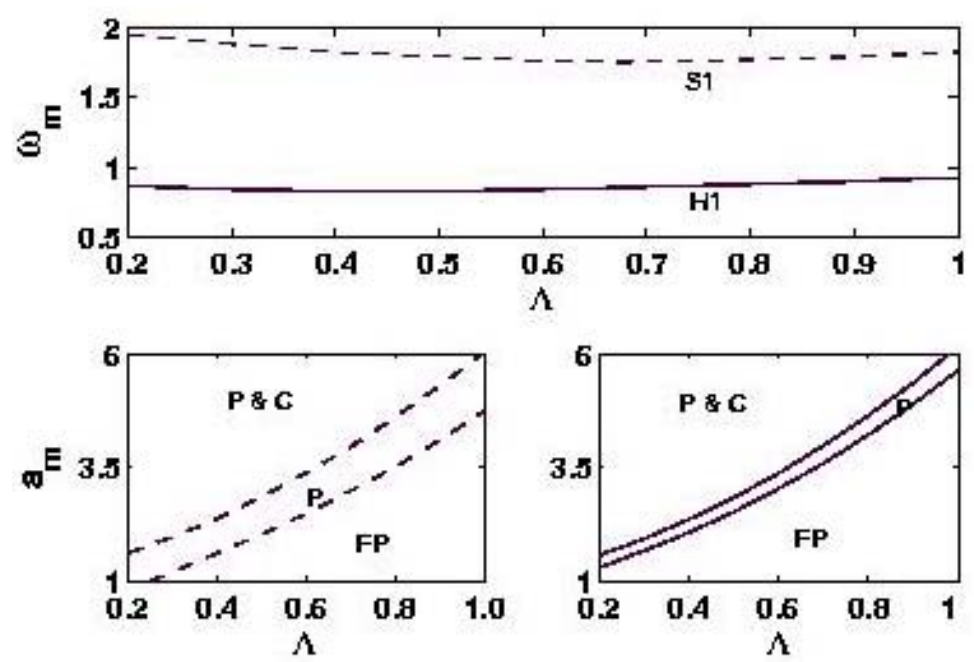

Fig.2. The minima of forcing amplitudes and the corresponding frequencies with damping for the first subharmonic (S1) and first harmonic (H1) response. The frequencies corresponding to the minima of the first subharmonic (S1) and the first harmonic (H1) tongues show a shallow minima (top row) with the damping coefficient $\Lambda$. The bottom row shows the threshold for instability of the subharmonically (left) and harmonically (right) driven pendulum as a function of $\Lambda$. The symbols 'FP', 'P' and 'P \& C' stand for fixed point, periodic, and mixed (periodic and chaotic) solutions, respectively.

Figure 2 shows the variation of the response frequency (top) and amplitude (bottom) as a function of the damping coefficient $\Lambda$. The frequencies corresponding to the minima of the subharmonic tongue $\mathrm{S} 1$ and harmonic tongue $\mathrm{H} 1$ show a shallow minima with damping. That means the tongues move first leftward and then rightward in the $a-\omega$ plane. However, the least value of the driving amplitude (bottom) shows monotonic increase as a function of the damping coefficient $\Lambda$. The least forcing is required always for exciting the pendulum subharmonically at a frequency corresponding to the minima of the subharmonic tongue $\mathrm{S} 1$.

\section{Nonlinear dynamics}

The nonlinear dynamics of a parametrically driven pendulum is governed by

$$
\ddot{X}+2 \Lambda \dot{X}+(1-a \cos \omega t) \sin X=0 .
$$


The above second order differential equation may be converted to a three-dimensional dynamical system given by

$$
\begin{aligned}
& \dot{X}=Y, \\
& \dot{Y}=-2 \Lambda Y-(1-a \cos \phi) \sin X, \\
& \dot{\phi}=\omega .
\end{aligned}
$$

The system is integrated by standard fourth order Runge-Kutta (RK4) method with randomly chosen initial conditions for the angular position $X$ and angular velocity $Y=\dot{X}$. We take the initial value of the phase $\phi=\omega t=0$. It is observed that the results of the linear Eq.(2.1), solved by RK4 and Floquet theory, are identical. The integration of the nonlinear pendulum equation shows ordered and chaotic dynamics. Periodic solutions are observed in the region labeled as 'P' (Fig.2, bottom). The upper curves in both the figures (bottom) are the boundaries for the period doubling bifurcation. A further increase in the driving amplitude $a$ leads to windows of periodic and chaotic solutions. They are labeled as 'P \& C' in Fig.2.
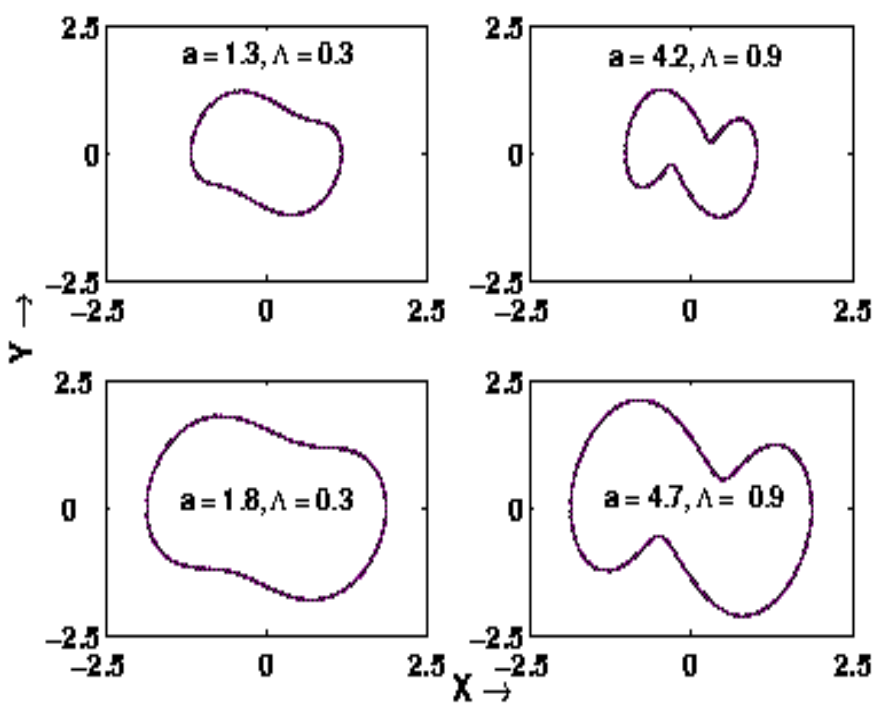

Fig.3. The phase portraits (in $X-Y$ plane) for the subharmonically driven pendulum for different values of $a^{\prime} s$ and $\Lambda^{\prime} s$. The driving frequency $\omega_{m}$ corresponds to the lowest point of the first subharmonic tongue (S1). The values of $\omega_{m}$ are 1.88 for $\Lambda=0.3$ and 1.79 for $\Lambda=0.9$.

Figure 3 shows the phase portraits of the subharmonically driven pendulum for different values of the damping coefficient and the forcing amplitude. The frequency is chosen corresponding to the lowest point of the first subharmonic tongue S1. If the forcing amplitude is raised keeping the damping fixed (both columns), the area of the limit cycles increases. This happens till the first period-doubling bifurcation occurs. On the other hand, the shape of the limit cycles at the onset of swing is distorted considerably, if the damping is increased (both rows). This leads to generation of many harmonics just above the onset of periodic swing of the pendulum. The interesting point is that the limit cycles in phase plane $X-Y$ maintain inversion symmetry.

Figure 4 shows a similar set of figures for harmonic driving. Several features are similar as for the case of subharmonic driving. The qualitative difference is the loss of inversion symmetry of the limit cycles 
in this case. The limit cycles for the harmonically forced damped pendulum never show the inversion symmetry. However, one may get another limit cycle for suitably chosen initial conditions, which may be related to the previous one by inversion symmetry. In addition, the first period-doubling bifurcation occurs for a relatively small increase in the forcing amplitude for the harmonically driven pendulum in comparison to the subharmonically driven situation (Fig.2, bottom). The inversion symmetry in the phase portraits of the subharmonically driven pendulum is connected with the temporal symmetries of the response. The subharmonic response displays $X(t \rightarrow t+T)=-X(t)$ symmetry for $T=2 \pi / \omega$, which is not true in the harmonically swing pendulum.

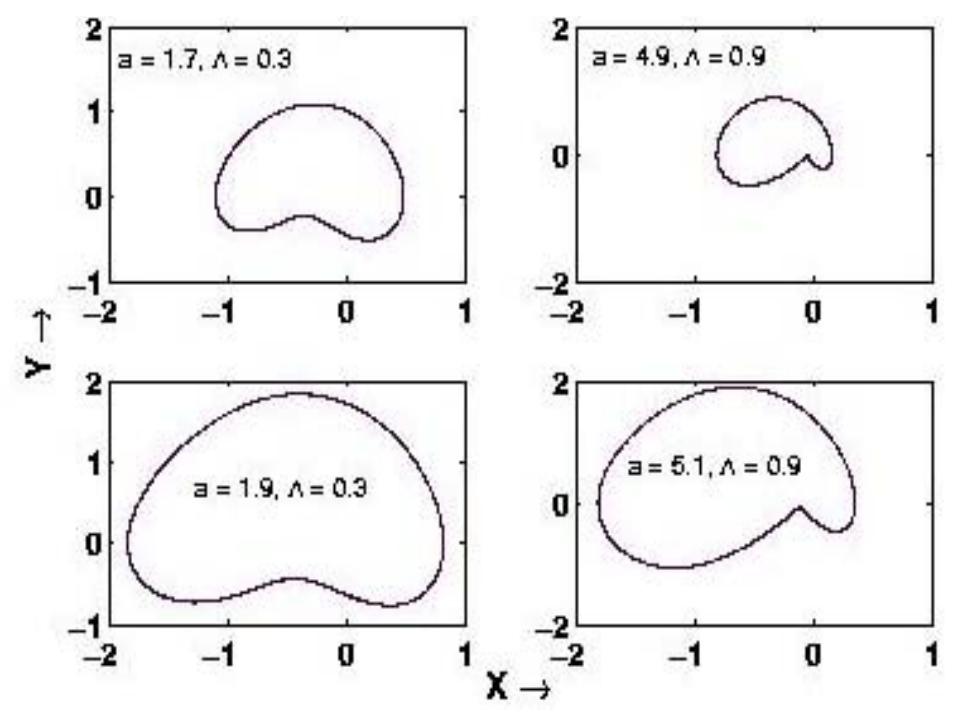

Fig.4. The phase portraits (in the $X-Y$ plane) for the harmonically driven pendulum for different values of $a^{\prime} s$ and $\Lambda^{\prime} s$. The driving frequency $\omega_{m}$ corresponds to the lowest point of the first harmonic tongue (H1). The values of $\omega_{m}$ are: 0.84 for $\Lambda=0.3$ and 0.90 for $\Lambda=0.9$.

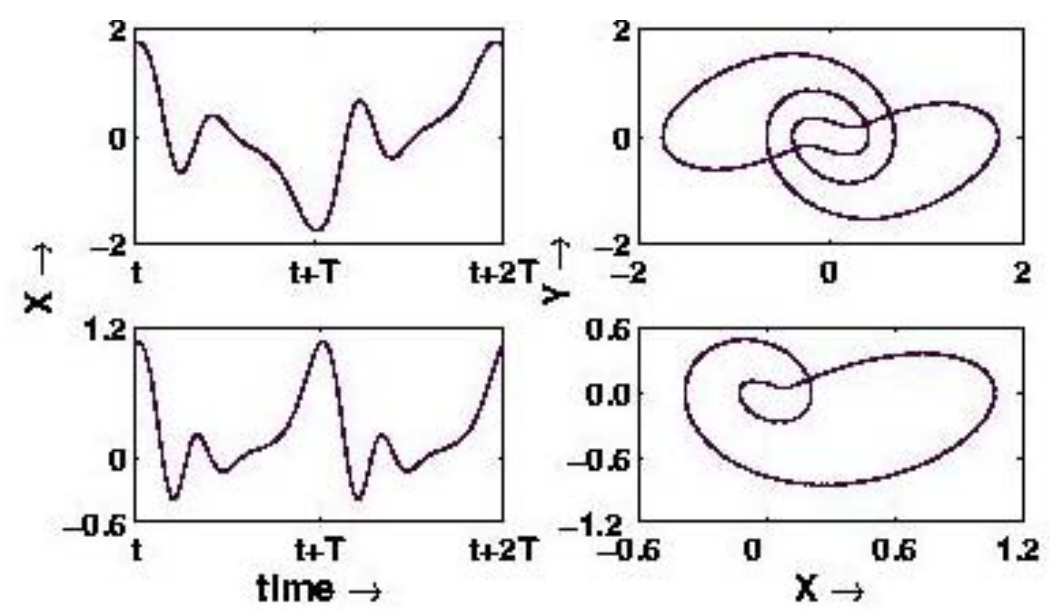

Fig.5. Time evolution of the angular position $X$ of the pendulum (left) and the trajectories in phase $(X-Y)$ plane (right) for frequencies corresponding to the minima of the second subharmonic (S2) tongue (top row) and the second harmonic (H2) tongue (bottom row), respectively. The forcing frequencies $\left(\omega_{m}\right)$ are: 0.55 (top row) and 0.42 (bottom row). The damping coefficient $\Lambda$ and the driving amplitude $a$ are 0.3 and 1.9 , respectively. 
The time dependence of the angular position $X(t)$ of the pendulum and the corresponding limit cycle are shown in Fig.5. The temporal evolution of the angular position of the subharmonically driven pendulum with a frequency corresponding to the lowest point of the second subharmonic (S2) tongue (upper row) shows three maxima/minima peaks (top left) in a period double the driving period. The corresponding limit cycle (top right) appears like period-3 solution. The correct stroboscopic map would give only one point instead of three, if the stroboscopic frequency is set half the driving frequency. The limit cycle also shows period-doubling bifurcation (not shown here), if the forcing amplitude is raised above the threshold for period doubling. The figures in the lower row are for frequency corresponding to the lowest point of the second harmonic tongue (H2). The angular position of the pendulum shows two maxima/minima peaks(bottom left) in a period equal to the driving period, and the corresponding limit cycle gives an illusion of period-doubling. An appropriate stroboscopic map would show again one point for this orbit.
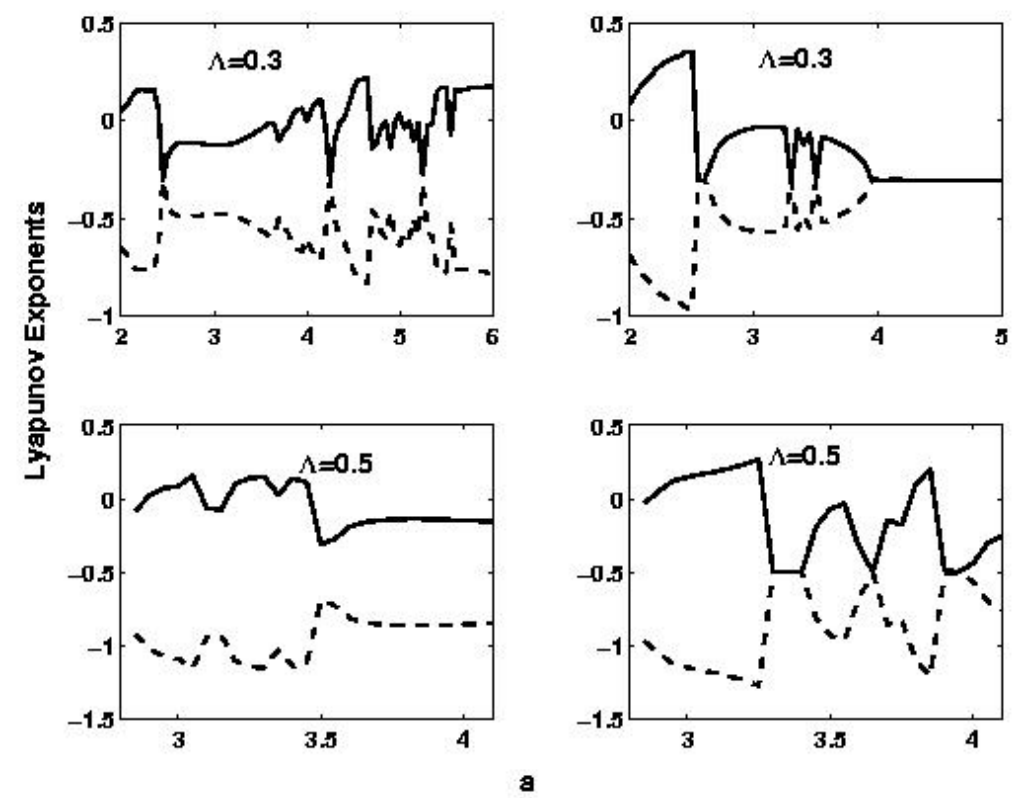

Fig.6. Lyapunov exponents as a function of the driving amplitude $(a)$ for the harmonically driven (left column) and subharmonically driven (right column) pendulum. The driving frequencies are chosen corresponding to the lowest points of the tongues labeled S1 and H1. The driving frequencies for the harmonic(left column) case are: 0.84 (top) and 1.84(bottom). For the case of subharmonic forcing (right column) they are: 1.88 (top) and 1.79 (bottom).

We observed windows of mixed solutions, where period-doubling, chaotic, and again periodic solutions appear (regions ' $\mathrm{P} \& \mathrm{C}$ ' in Fig.2) above a threshold (upper curves of the bottom figures). To determine the windows of chaotic solutions, the computation of Lyapunov exponents is essential. We set the driving frequency $\omega$ at a value corresponding to the least point of the first subharmonic or the first harmonic tongue and compute all the Lyapunov exponents using the method proposed by Wolf et al. 1985). For very small values of $\Lambda(<0.01)$, the largest Lyapunov exponent becomes smaller and computation becomes difficult. For the values of $\Lambda(\geq 0.1)$, the method used gives very accurate results. We have shown Lyapunov exponents for $\Lambda=0.3$ and 0.5 . Very long signals (more than three million time steps) are used for the computation of Lyapunov exponents. One of the exponent is always zero owing to the presence of periodic driving. Figure 6 displays two non-zero Lyapunov exponents for the harmonically (left column) and subharmonically (right column) driven pendulum for different values of the damping coefficient. As one out 
of three Lyapunov exponents is always zero, the remaining two show mirror symmetry about the $a$-axis. The sum of all Lyapunov exponents is equal to $-\Lambda$ for the damped pendulum. The values of $a$ for which, the largest Lyapunov exponent becomes positive would make the dynamics of the parametrically driven pendulum chaotic. For smaller damping coefficients, the number of windows is large and their widths are small. With an increase in the damping coefficient, the number of windows becomes smaller with larger widths. The driven pendulum shows periodic behavior at large amplitude forcing for larger damping coefficients.
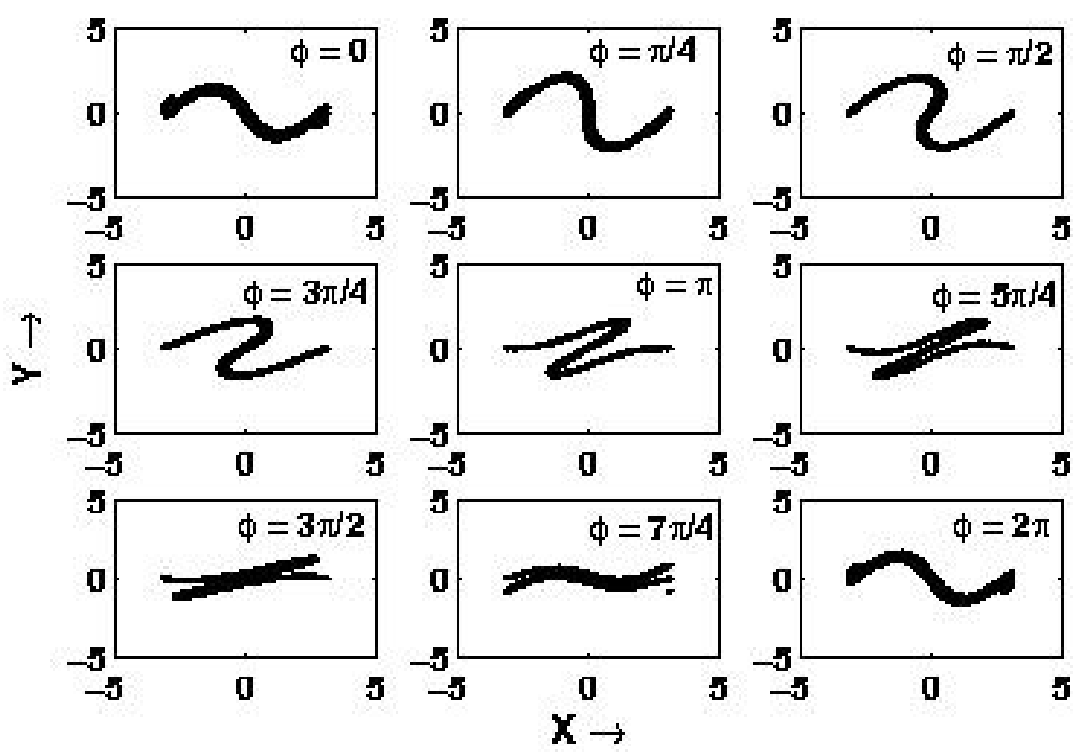

Fig.7. Stroboscopic maps for chaotic state of the subharmonically driven pendulum for different values of the phase $\phi=\omega t$. The driving frequency $\omega_{m}$, the driving amplitude $a$ and the damping coefficient $\Lambda$ are $1.88,2.3$ and 0.3 , respectively. All the maps show inversion symmetry in the $X$ - $Y$ plane, and they repeat after a phase of $2 \pi$.

We now investigate numerically the stroboscopic maps (Baker and Gollub, 1990) for chaotic state of the parametrically driven pendulum. We consider various values of the phase $\phi=\omega t$ to construct the stroboscopic maps. The strobe frequency is set at the driving frequency $\omega$. The maps at a given value of $\phi$ are the collections of all phase points in $X-Y$ plane at an interval of time $T=2 \pi / \omega$. Figure 7 displays stroboscopic maps of the subharmonically driven pendulum for different phases at equal interval of $\pi / 4$ starting from $\phi=0$. Each of the strange attractors shows inversion symmetry in the $X-Y$ plane. This may be connected with the $X(t+T)=-X(t)$ symmetry of subharmonic response. Each of the stroboscopic maps displaying a part of the strange attractor appears connected with its neighbors by stretching in two directions. The shape of the strange attractors repeats after a phases change of $2 \pi$.

Figure 8 displays stroboscopic maps for different values of the phase $\phi$ for the harmonically driven pendulum. These maps display inversion symmetry in the $X$ - $Y$ plane, though the limit cycles (shown in Fig.4) do not. This is qualitatively different from strange attractors of a non-parametrically driven pendulum (Baker and Gollum, 1990) which does not show inversion symmetry in the stroboscopic map. In addition, the stroboscopic maps for different $\phi$ do not show symmetry about the phase $\phi=\pi$ for the parametrically forced pendulum as it is observed in non-parametric forcing. The strange attractors corresponding to different phases of a harmonically driven pendulum appear to be connected by stretching and rotation. 

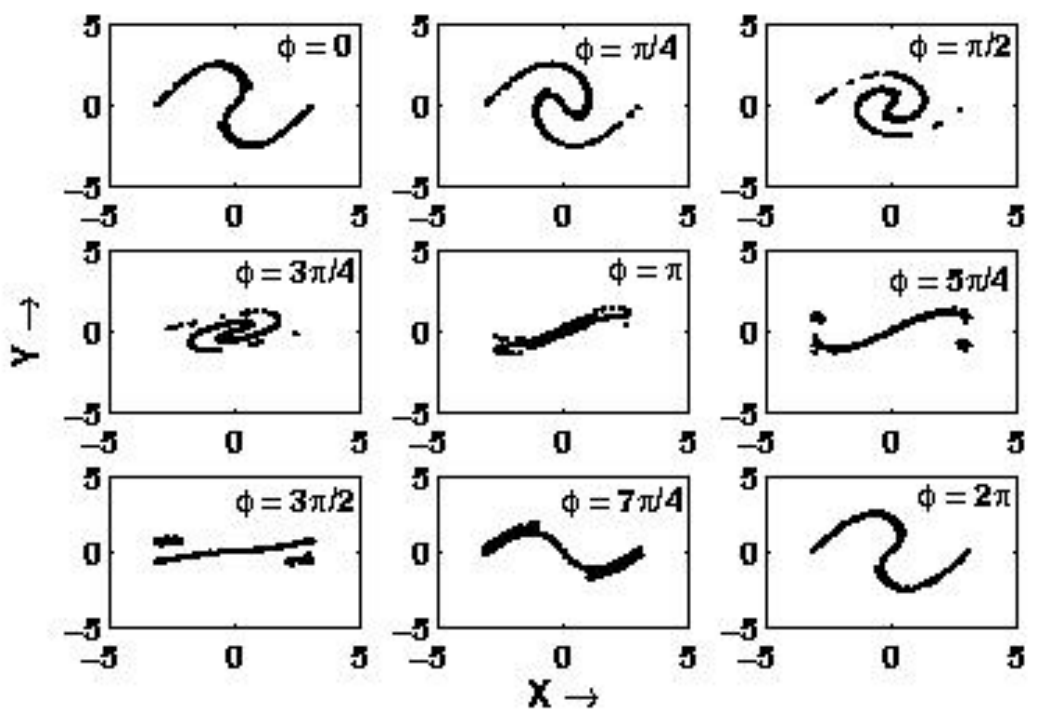

Fig.8. Stroboscopic maps for chaotic state of the harmonically driven pendulum for different values of the phase $\phi=\omega t$. The driving frequency $\omega_{m}$, the deriving amplitude $a$ and the damping coefficient $\Lambda$ are $0.84,2.3$ and 0.3 , respectively. All the maps show inversion symmetry in the $X-Y$ plane, and they repeat after a phase of $2 \pi$.

\section{Conclusions}

We have presented here a systematic study of ordered and chaotic states of a parametrically driven pendulum with viscous damping. Lyapunov exponents against the forcing amplitude $(a)$ show that chaotic windows become fewer and wider with increasing damping. The ordered states of the subharmonically driven pendulum show inversion symmetry in the phase plane, while those for the harmonically driven pendulum do not. However, the pendulum becomes chaotic only via period-doubling bifurcations. The strange attractors of a pendulum driven either harmonically or subharmonically always show inversion symmetry in the phase plane, which is in contrast with the strange attractors observed in a nonparametrically driven pendulum.

\section{Acknowledgements}

The authors are thankful to Prof. H.P. Majumder for his advice to publish this work.

\section{Nomenclature}

$$
\begin{aligned}
\mathcal{A} & - \text { diagonal matrix } \\
a & - \text { forcing amplitude } \\
\mathcal{B} & - \text { tridiagonal matrix } \\
F P & - \text { fixed point solution } \\
H n & -n^{\text {th }} \text { harmonic response } \\
P & - \text { periodic solution }
\end{aligned}
$$

$P$ and $C$ - periodic and chaotic solution

$S n-n^{\text {th }}$ subharmonic response

$T$ - time period of a signal

$X(t)$ - angular position of the pendulum at time $t$

$\Lambda$ - damping term 


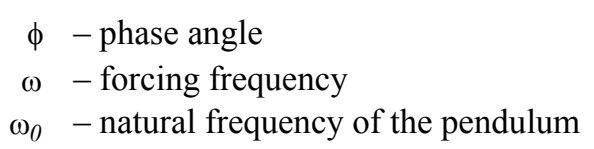

\section{References}

Arscott F.M. (1964): Periodic Differential Equations. - Pergamon.

Baker G.L. and Gollub J.P. (1990): Chaotic Dynamics: an Introduction. - Cambridge University Press.

Bartuccelli M.V., Gentile G. and Georgiou K.V. (2001): On the dynamics of a vertically driven damped planar pendulum. - Proc. R. Soc. Lond A, vol.457, pp.3007-3022.

Bender C.M. and Orszag S.A. (1978): Advanced Mathematical Methods for Scientists and Engineers. - McGrew Hill.

Faraday M. (1831): On a peculiar class of acoustical figures; and on certain forms assumed by groups of particles upon vibrating elastic surfaces. - Trans. R. Soc. Lond. A, vol.121, pp.299-340.

Jing Z. Jing and Yang J. (2006): Complex dynamics in pendulum equation with parametric and external excitations I. Int. J. Bifurcation and Chaos, vol.16, No.10, pp.2887-2902.

Jordan D.W. and Smith P. (1977): Nonlinear Ordinary Differential Equations. - Oxford: Clarendon Press.

Kumar K. (1996): Linear theory of faraday instability in viscous liquids. - Proc. R. Soc. A, vol.452, pp.1113-1126.

Landau L.D. and Lifschitz E.M. (1976): Mechanics, 3rd edn.. - Oxford: Pergamon.

Leven R.W., Pompe B., Wilke C., and Koch B.P. (1985): Experiments on periodic and chaotic motions of a parametrically forced pendulum. - Physica D, vol.16, pp.371-384.

McLaughlin J. (1981): Period-doubling bifurcations and chaotic motions for a parametrically forced pendulum. Journal of Statistical Physics, vol.24, pp.375-388.

Starrett J. and Tagg R. (1995): Control of a chaotic parametrically driven pendulum. - Phys. Rev. Lett., vol.74, No.11, pp.1974-1977.

Van de Water W., Hoppenbrouwers M., and Christiansen F. (1991): Unstable periodic orbits in the parametrically excited pendulum. - Phys. Rev. A, vol. 44, No.10, pp.6388-6398.

Wolf A., Swift J.B., Swinney H.L. and Vastano J.A. (1985): Determining Lyapunov exponents from a time series. Physica D, vol.16, No.3, pp.285-317. 